\title{
Risk Assessment Using Early Quantitative Chest CT Parameters for the Severity of COVID-19
}

\author{
Xun Ding ${ }^{1}$, Jia Xu ${ }^{1}$, Haibo $\mathrm{Xu}^{1}{ }^{1}$ Jun Zhou ${ }^{1,{ }^{*}}$ and Qingyun Long ${ }^{1, * *}$ \\ ${ }^{1}$ Department of Radiology, Zhongnan Hospital of Wuhan University, Wuhan University, 430071, Wuhan, China \\ "Corresponding author: Department of Radiology, Zhongnan Hospital of Wuhan University, Wuhan University, 430071, Wuhan, China. Email: zhoujunzn@126.com \\ "* Corresponding author: Department of Radiology, Zhongnan Hospital of Wuhan University, Wuhan University, 430071, Wuhan, China. Email: longqy2005@126.com \\ Received 2020 September 22; Revised 2021 June 07; Accepted 2021 June 09.
}

\begin{abstract}
Background: Today, the outbreak of coronavirus disease 2019 (COVID-19) is known as a public health emergency by the World Health Organization (WHO). Therefore, risk assessment is necessary for making a correct decision in disease management.

Objectives: This study aimed to assess the risk of progression to the critical stage in COVID-19 patients, based on the early quantitative chest computed tomography (CT) parameters.

Patients and Methods: In this case-control study, 39 laboratory-confirmed critical or expired COVID-19 cases (critical group), as well as 117 laboratory-confirmed COVID-19 patients including mild, moderate, and severe cases (non-critical group), were enrolled. Seven quantitative CT parameters, representing the lung volume percentages at different density intervals, were automatically calculated, using the artificial intelligence (AI) algorithms. Multivariable-adjusted logistic regression models, based on the quantitative CT parameters, were established to predict the adverse outcomes (critical vs. non-critical). The predictive performance was estimated using the receiver operating characteristic (ROC) curve analysis and by measuring the area under the ROC curve (AUC). The quantitative CT parameters in different stages were compared between the two groups.

Results: No significant differences were found between the two groups regarding the lung volume percentages at different density intervals within 0 - 4 days $(\mathrm{P}=0.596-0.938)$; however, this difference began to become significant within 5 - 9 days and persisted even after one month. Overall, the quantitative CT parameters could well predict the severity of COVID-19. The lung volume percentage of -7 Hounsfield units (-7 HUs) had the largest crude odds ratio (OR:1.999; 95\% CI, 1.453 2.750; $\mathrm{P}<0.001$ ) and adjusted OR (adjusted OR: 1.768; 95\% CI, 1.114 2.808; $\mathrm{P}=0.016)$. The lung volume percentage of $-6 \mathrm{HU}$ showed the best predictive performance with the largest AUC of 0.808 ; the cutoff value of $5.93 \%$ showed $71.79 \%$ sensitivity and $84.62 \%$ specificity.

Conclusion: Early quantitative chest CT parameters can be measured to assess the risk of progression to the critical stage of COVID19 ; this is of critical importance in the clinical management of this disease.
\end{abstract}

Keywords: Quantitative CT, COVID-19, Critical, Artificial Intelligence

\section{Background}

Today, coronavirus disease 2019 (COVID-19), caused by severe acute respiratory syndrome coronavirus 2 (SARSCoV-2), has spread around the world. As of May 30, 2020, a total of 6,025,764 confirmed cases of COVID-19 were reported worldwide, with 368,404 deaths (6.1\%). Patients with COVID-19 may have different outcomes, and therefore, they need different levels of clinical care. Most patients with COVID-19 have mild symptoms or no symptoms, while some patients can progress to severe pneumonia rapidly, leading to acute respiratory distress syndrome (ARDS), multiple organ dysfunction syndrome (MODS), or even death.
Currently, several clinical and laboratory indicators are used to assess the severity of COVID-19 in patients with infectious pneumonia and to predict their prognosis (1-5). However, some clinical and laboratory tests are not accurate enough. Also, most of these tests are invasive and may cause iatrogenic infections. Besides, scoring systems, such as the Acute Physiology and Chronic Health Evaluation (APACHE-II), are often subjective and time-consuming and may not be conducive to a timely clinical intervention for COVID-19. Computed tomography (CT) plays an important role in screening, diagnosis, evaluation, and followup of patients with COVID-19 (6-9). However, the CT evaluation of patients with COVID-19 is often semi-quantitative 
and greatly affected by subjective factors related to the radiologist. Therefore, it cannot accurately quantify the CT features or quantitatively assess the severity of disease.

With the rise of artificial intelligence (AI), this branch has been incorporated in different medical fields, such as risk and prognosis prediction of lung cancer and ARDS (10, 11). Considering the fast and non-invasive nature of CT examination, besides the objectivity and efficiency of AI, if AIderived features, obtained from CT in the early stages of COVID-19, can be used to assess the risk of progression to the critical stage, they can be highly beneficial in clinical interventions for the disease. However, there are few reports on the application of AI in the evaluation of COVID-19 $(12,13)$.

\section{Objectives}

In this study, we aimed to investigate the value of AIderived quantitative CT parameters in assessing the risk of progression to the critical stage of COVID-19.

\section{Patients and Methods}

\subsection{Study Design and Participants}

This case-control study was performed on hospitalized patients with laboratory-confirmed COVID-19 pneumonia, who underwent CT examinations on admission at a single hospital from January 2020 to March 2020. All patients were discharged or expired before the date of data collection. They were divided into two groups based on the clinical outcomes: (1) critical group (case), including critical and expired patients; and (2) non-critical group (control) including mild, moderate, and severe patients. The controls were matched for age and comorbidities with the case group and enrolled at a case-control ratio of 1: 3 . All patients in the case and control groups received standard institutional care for COVID-19 pneumonia during hospitalization. This retrospective study was approved by the local ethics committee, and the need to obtain informed consent was waived.

\subsection{Data Collection}

The demographic, clinical, laboratory, and CT data of the case and control groups were retrieved from the electronic medical records and the picture archiving and communication system (PACS) in a hospital form via standardized collection.

\subsection{CT Protocol}

CT imaging was performed at the end of expiration, while the patient was lying in a supine position with both arms raised. Two CT scanners were used for 156 patients in this study. The CT parameters in the GE Discovery CT750 HD system were as follows: (1) reconstructed slice thickness, $1.25 \mathrm{~mm}$; (2) slice gap, $1.25 \mathrm{~mm}$; (3) tube voltage, $120 \mathrm{kV}$; (4) automatic tube current modulation, and display field of view (DFOV), $40.0 \times 46.6 \mathrm{~cm}$. Also, the CT parameters in the siemens somatom definition system were as follows: (1) reconstructed slice thickness, $1.0 \mathrm{~mm}$; (2) slice gap, $1.0 \mathrm{~mm}$; (3) tube voltage, $120 \mathrm{kV}$; (4) automatic tube current modulation, and DFOV, $36.8 \times 42.9 \mathrm{~cm}$.

\subsection{Quantitative Chest CT Analysis}

A quantitative CT system (YITU-CT-Lung, YITU Healthcare Technology Co., Ltd., China) was used to quantitatively analyze the pulmonary involvement of COVID-19 (Figure 1). The AI algorithms were used to automatically calculate seven quantitative parameters, representing the lung volume percentages in density intervals of $-1000 \sim-700 \mathrm{HU}$ (HU-1), -700 -600 HU(HU-2), -600 -500 HU(HU-3), -500 $\sim-300 \mathrm{HU}(\mathrm{HU}-4),-300 \sim-200 \mathrm{HU}(\mathrm{HU}-5),-200 \sim 60 \mathrm{HU}$ (HU-6), and $60 \sim 1000 \mathrm{HU}(\mathrm{HU}-7)$.

The CT scans were categorized according to the interval from the onset of initial symptoms until the CT scan: stage 1 ( 0 - 4 days; $n=43$ ); stage 2 ( 5 - 9 days; $n=75$ ); stage 3 (10 - 14 days; $n=79)$; stage 4 (15 - 21 days; $n=83)$; stage 5 (22 28 days; $\mathrm{n}=80$ ); and stage 6 ( $>28$ days; $\mathrm{n}=110)(6,8)$. The quantitative CT parameters in different stages were compared between the two groups.

\subsection{Statistical Analysis}

Statistical analyses were performed in SPSS version 23.0 (IBM Inc., Chicago, IL, USA) and GraphPad Prism version 7.0 (GraphPad Software Inc., USA). Continuous variables were first examined using Kolmogorov-Smirnov test for normality. They are presented as mean \pm standard deviation (SD) and compared between the groups using Mann-Whitney $U$ test. Categorical variables are presented as number (percentage of total) and compared between the groups using chi-square test or Fisher's exact test.

A binary logistic regression analysis was performed to explore the relationship between the quantitative CT parameters and the severity of COVID-19. A multivariableadjusted logistic regression model, adjusted for sex, age, white blood cell (WBC) count, alanine aminotransferase (ALT), creatinine, procalcitonin (PCT), creatine kinase 

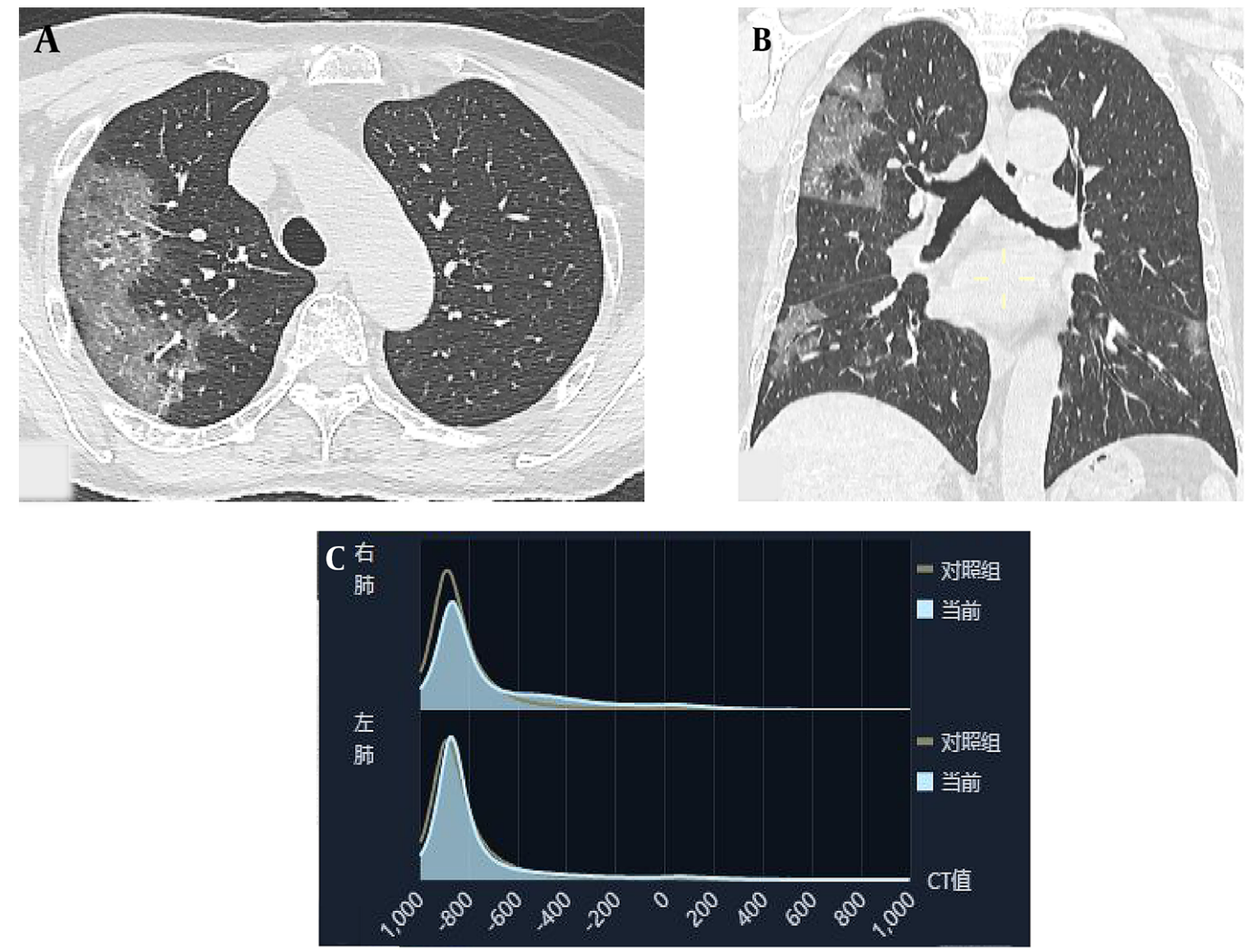

\begin{tabular}{|c|c|c|c|c|c|c|}
\hline (HU) & $\begin{array}{l}\text { W肺 } \\
(\%)\end{array}$ & $\begin{array}{l}\text { 在肺 } \\
(\%)\end{array}$ & $\begin{array}{l}\text { 古肺 } \\
(\%)\end{array}$ & $\begin{array}{l}\text { 双螬 } \\
\left(\mathrm{cm}^{3}\right)\end{array}$ & $\begin{array}{l}\text { 右触 } \\
\left(\mathrm{cm}^{3}\right)\end{array}$ & \\
\hline$-1000 \sim-700$ & 70.52 & 64.08 & 77.58 & 2553.00 & 1213.89 & 1. \\
\hline$-700 \sim-600$ & 5.84 & 6.55 & 5.05 & 211.30 & 124.06 & 8 \\
\hline$-600 \sim-500$ & 4.20 & 5.36 & 2.92 & 151.95 & 101.55 & 5 \\
\hline$-500 \sim-300$ & 5.95 & 8.11 & 3.58 & 215.35 & 153.63 & 6 \\
\hline$-300--200$ & 1.75 & 2,39 & 1.05 & 63.48 & 45.30 & 14 \\
\hline$-200 \sim 60$ & 3.61 & 4.82 & 2.28 & 130.55 & 91.21 & 3) \\
\hline $60 \sim 1000$ & 2.73 & 3.35 & 2.05 & 98.94 & 63.51 & 3 \\
\hline all & 100.00 & 100.00 & 100.00 & 3620.31 & 1894.22 & 1 \\
\hline
\end{tabular}

Figure 1. AI-based quantitative chest CT analysis of a patient with COVID-19. A, Axial CT image; B, Coronal CT image; C, Histogram of total lung density; and D, Distribution table of total lung volume (AI, artificial intelligence; COVID-19, Coronavirus disease 2019). 
isoenzyme MB (CK-MB), and D-dimer, based on the quantitative CT parameters, was established to predict critical cases (critical vs. non-critical). Moreover, the predictive performance was estimated using the receiver operating characteristic (ROC) curve and by measuring the area under the ROC curve (AUC). When P-value was less than 0.05, the difference was considered to be statistically significant.

\section{Results}

\subsection{Clinical and Laboratory Data}

The most common symptoms at the onset of disease were fever (78.8\%) and dry cough (41.7\%), followed by fatigue (34.0\%) and chest distress (24.4\%). Although the incidence of these symptoms was higher in the critical group than in the non-critical group, no significant difference was found between the two groups (Table 1). Hypertension was the most common comorbidity (28.8\%), followed by diabetes (16.7\%) and cardiovascular disease (6.4\%). Although the incidence of hypertension, diabetes, and cardiovascular disease in the critical group was higher than the noncritical group, no significant difference was found between the groups (Table 1 ).

The incidence rate of leukocytosis and elevated levels of ALT, PCT, CK-MB, and D-dimer were higher in the critical group than in the non-critical group, and there was a significant difference between the groups (Table 1). Although the incidence of creatinine increase in the critical group was higher than the non-critical group, no significant difference was found between the two groups (Table 1).

\subsection{Quantitative Chest CT Analysis in Different Stages}

A total of $470 \mathrm{CT}$ scans ( mean $=3.0 ; \mathrm{SD}=1.6$ ) were collected from 156 patients. In stage 1 , the lung volume percentage in different density intervals was not significantly different between the two groups ( $\mathrm{P}=0.596$ - 0.938) (Figure 2 ). In other stages, the lung volume percentage in different density intervals was significantly different between the two groups $(\mathrm{P}<0.05)$. However, in stage 2 and stage 4 , the lung volume percentage was not significantly different between the two groups at the HU-2 interval $(\mathrm{P}=0.158$ and $\mathrm{P}=0.371$, respectively) (Figure 2 ).

4.3. Multivariable-Adjusted Associations Between Quantitative CT Parameters and COVID-19 Severity Based on The Binary Logistic Regression Model

We found that the quantitative CT parameters were related to the outcomes of patients with COVID-19 (Table 2).
Based on the binary regression analysis, the lung volume percentage in HU-1 was a protective factor for the adverse outcomes of COVID-19, with a crude odds ratio(OR) of 0.931 (95\% CI, 0.905 0.958; $\mathrm{P}<0.0001$ ) and an adjusted OR of 0.953 (95\% CI, $0.918 \sim 0.990 ; \mathrm{P}=0.013$ ). The lung volume percentages in other HU intervals were risk factors for the adverse outcomes of COVID-19.

The crude OR for HU-2 was 1.162 (95\% CI, 1.039 1.300; P $=0.008$ ) with an adjusted OR of 1.027 (95\% CI, 0.875 1.206; $\mathrm{P}=0.743$ ); the crude OR for HU-3 was 1.404 (95\% CI, 1.199 1.646; $\mathrm{P}<0.0001$ ) with an adjusted OR of 1.157 (95\% CI, 0.933 $\sim 1.436 ; \mathrm{P}=0.184$ ); the crude OR for HU-4 was 1.281 (95\% CI, 1.157 1.419; $\mathrm{P}<0.0001)$ with an adjusted OR of 1.159 (95\% $\mathrm{CI}, 1.011 \sim 1.306 ; \mathrm{P}=0.035)$; and the crude OR for HU-5 was 1.888 (95\% CI,1.454 2.451; $\mathrm{P}<0.0001$ ) with an adjusted $\mathrm{OR}$ of 1.592 ( $95 \% \mathrm{CI}, 1.141 \sim 2.222 ; \mathrm{P}=0.006)$.

Also, the crude OR for HU-6 was 1.303 (95\% CI, 1.170 1.452) with an adjusted OR of 1.237 (95\% CI, $1.080 \sim 1.415 ; \mathrm{P}$ $=0.002$ ); the crude OR of HU-7 was 1.999 (95\% CI, 1.453 2.750; $\mathrm{P}<0.0001$ ) with an adjusted OR of 1.768 (95\% CI, 1.114 $\sim$ 2.808; P = 0.016); the crude OR of HU-2+HU-3 was 1.127 (95\% CI, $1.053 \sim 1.206$; P $=0.001$ ) with an adjusted OR of 1.035 (95\% CI, $0.943 \sim 1.136 ; \mathrm{P}=0.471$ ); and the crude OR of HU-4+HU-5 was 1.198 (95\% CI, 1.113 1.290; $\mathrm{P}<0.0001$ ) with an adjusted $\mathrm{OR}$ of 1.125 (95\% CI, $1.020 \sim 1.240 ; \mathrm{P}=0.018$ ) (Table 2).

\subsection{Predictive Performance of Quantitative Chest CT Scan for COVID-19 Severity}

The ROC curve was used to analyze the predictive performance of quantitative chest CT parameters for COVID-19 severity on admission. The AUC range of quantitative chest CT was $0.688 \sim 0.808(\mathrm{P}<0.0001)$. The lung volume percentage in HU-6 showed the largest AUC (AUC: 0.808; 95\% CI, $0.716 \sim 0.900 ; \mathrm{P}<0.0001)$, and the cutoff value of 5.93 had $71.79 \%$ sensitivity and $84.62 \%$ specificity (Figure 3 and Table 3).

\section{Discussion}

To the best of our knowledge, few studies have used non-invasive quantitative CT measurements to predict the severity of COVID-19 $(12,14)$. For the first time, we used the lung volume percentages in different density intervals to assess the risk of progression to the critical stage of COVID19. According to different CT values, we divided the entire lung into seven density intervals and used AI for automatic quantification. Our results showed that all quantitative CT 


\begin{tabular}{|c|c|c|c|c|}
\hline Variables & All patients $(n=156)$ & Critical $(n=39)$ & Non-critical $(\mathbf{n}=117)$ & P-value \\
\hline \multicolumn{5}{|l|}{ Gender } \\
\hline Female & $77(49.4)$ & $11(28.2)$ & $66(56.4)$ & - \\
\hline Age $(y)$ & $59 \pm 15$ & $63 \pm 14$ & $58 \pm 15$ & 0.059 \\
\hline Male & $61 \pm 16$ & $63 \pm 15$ & $60 \pm 16$ & 0.580 \\
\hline Female & $57 \pm 14$ & $65 \pm 10$ & $56 \pm 14$ & 0.040 \\
\hline \multicolumn{5}{|l|}{ Initial symptoms } \\
\hline Fever & $123(78.8)$ & $34(87.2)$ & $89(76.1)$ & 0.141 \\
\hline Low-grade fever $\left(37.3-38.0^{\circ} \mathrm{C}\right)$ & $61(39.1)$ & $10(25.6)$ & $51(43.6)$ & 0.047 \\
\hline Moderate-grade fever $\left(38.1-39.0^{\circ} \mathrm{C}\right)$ & $52(33.3)$ & $22(56.4)$ & $30(25.6)$ & $<0.0001$ \\
\hline High-grade fever $\left(>39.0^{\circ} \mathrm{C}\right)$ & $9(5.8)$ & $2(5.1)$ & $7(6.0)$ & 1.000 \\
\hline Expectoration & $17(10.9)$ & $2(5.1)$ & $15(12.8)$ & 0.299 \\
\hline Throat pain & $6(3.8)$ & $1(2.5)$ & $5(4.3)$ & 0.978 \\
\hline Chest distress & $38(24.4)$ & $12(30.8)$ & $26(22.2)$ & 0.282 \\
\hline Dyspnea & $26(16.7)$ & $12(30.8)$ & $14(12.0)$ & 0.006 \\
\hline Fatigue & $53(34.0)$ & $18(46.2)$ & $35(29.9)$ & 0.064 \\
\hline Nausea and vomiting & $5(3.2)$ & $1(2.6)$ & $4(3.4)$ & 1.000 \\
\hline Abdominal pain and diarrhea & $18(11.5)$ & $3(7.7)$ & $15(12.8)$ & 0.563 \\
\hline Myalgia & $23(14.7)$ & $6(15.4)$ & $17(14.5)$ & 0.896 \\
\hline Headache & $9(5.8)$ & $2(5.1)$ & $7(6.0)$ & 1.000 \\
\hline Dizziness & $3(1.9)$ & $0(0.0)$ & $3(2.6)$ & 0.574 \\
\hline Palpitation & $3(1.9)$ & $1(2.6)$ & $2(1.7)$ & 1.000 \\
\hline \multicolumn{5}{|l|}{ Comorbidities } \\
\hline Hypertension & $45(28.8)$ & $14(35.9)$ & $31(26.5)$ & 0.262 \\
\hline Cardiovascular disease & $10(6.4)$ & $4(10.3)$ & $6(5.1)$ & 0.450 \\
\hline \multicolumn{5}{|l|}{ Laboratory markers } \\
\hline White blood cell count (WBC) $(109 / \mathrm{L})$ & $6.41 \pm 3.94$ & $9.36 \pm 5.29$ & $5.43 \pm 2.77$ & $<0.0001$ \\
\hline$<4$ & $39(25.0)$ & $4(10.3)$ & $35(29.9)$ & $<0.0001$ \\
\hline $4-10$ & $102(65.4)$ & $24(61.5)$ & $78(66.7)$ & - \\
\hline$>10$ & $15(9.6)$ & $11(28.2)$ & $4(3.4)$ & - \\
\hline Alanine aminotransferase (ALT) (U/L) & $37.46 \pm 34.01$ & $58.08 \pm 44.45$ & $30.59 \pm 26.60$ & $<0.0001$ \\
\hline$\leq 50$ & $127(81.4)$ & $23(59.0)$ & $104(88.9)$ & $<0.0001$ \\
\hline$>50$ & $29(18.6)$ & $16(41.0)$ & $13(11.1)$ & - \\
\hline Creatinine $(\mu \mathrm{mol} / \mathrm{L})$ & $71.34 \pm 23.67$ & $79.58 \pm 29.60$ & $68.60 \pm 20.76$ & 0.035 \\
\hline$\leq 104$ & $144(92.3)$ & $33(84.6)$ & $111(94.9)$ & 0.083 \\
\hline$>104$ & $12(7.7)$ & $6(15.4)$ & $6(5.1)$ & - \\
\hline \multicolumn{5}{|l|}{ Procalcitonin (PCT) $(\mathbf{n g} / \mathbf{m L})$} \\
\hline$<0.05$ & $74(59.2)$ & $9(23.1)$ & $65(75.6)$ & $<0.0001$ \\
\hline$\geq 0.05$ & $51(40.8)$ & $30(76.9)$ & $21(24.4)$ & - \\
\hline Creatine kinase-MB (CK-MB) (U/L) & $15.81 \pm 13.29$ & $23.03 \pm 20.80$ & $12.89 \pm 6.84$ & $<0.0001$ \\
\hline$\leq 25$ & $118(89.4)$ & $28(73.7)$ & $90(95.7)$ & 0.001 \\
\hline$>25$ & $14(10.6)$ & $10(26.3)$ & $4(4.3)$ & - \\
\hline D-dimer (ng/mL) & $2596.32 \pm 6972.42$ & $6390.00 \pm 10983.84$ & $1187.24 \pm 3919.41$ & $<0.0001$ \\
\hline$\leq 500$ & $96(66.7)$ & $13(33.3)$ & $83(79.0)$ & $<0.0001$ \\
\hline$>500$ to $\leq 1000$ & $13(9.0)$ & $7(17.9)$ & $6(5.7)$ & - \\
\hline$>1000$ & $35(24.3)$ & $19(48.7)$ & $16(15.2)$ & - \\
\hline Time from the initial symptoms until admission (d) & $10.8 \pm 7.3$ & $10.9 \pm 5.6$ & $10.7 \pm 7.8$ & 0.308 \\
\hline
\end{tabular}




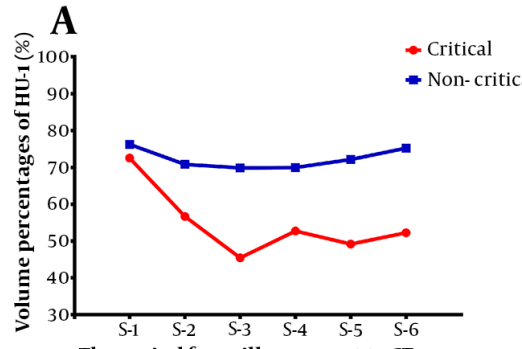

The period from illness onset to CT scans
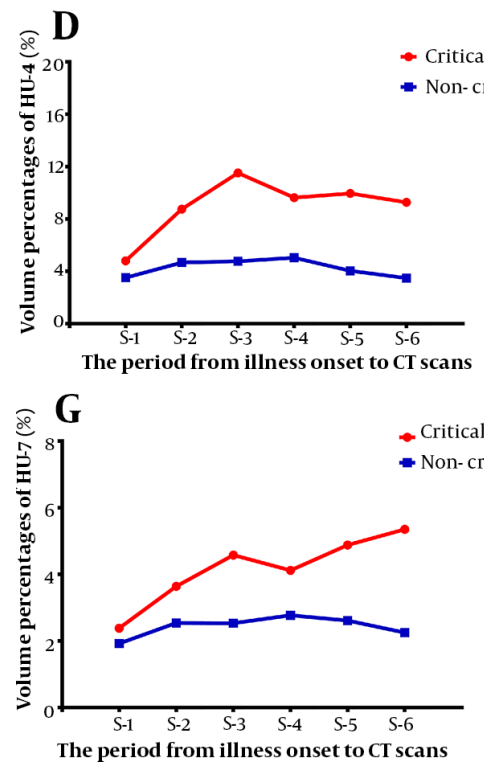
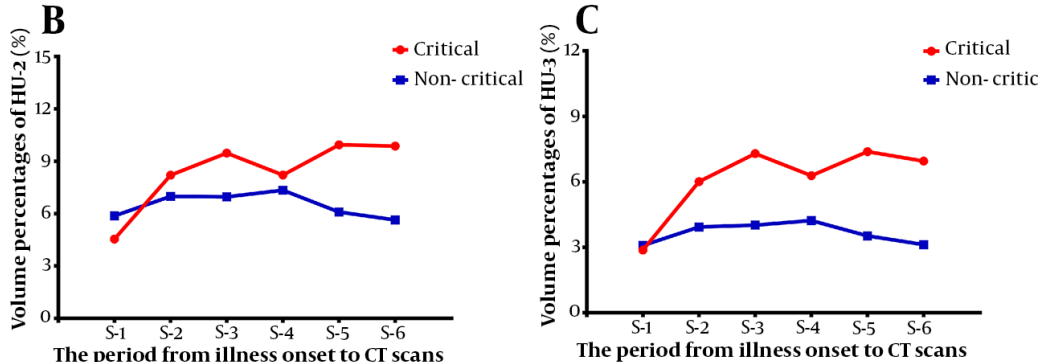

The period from illness onset to CT scans
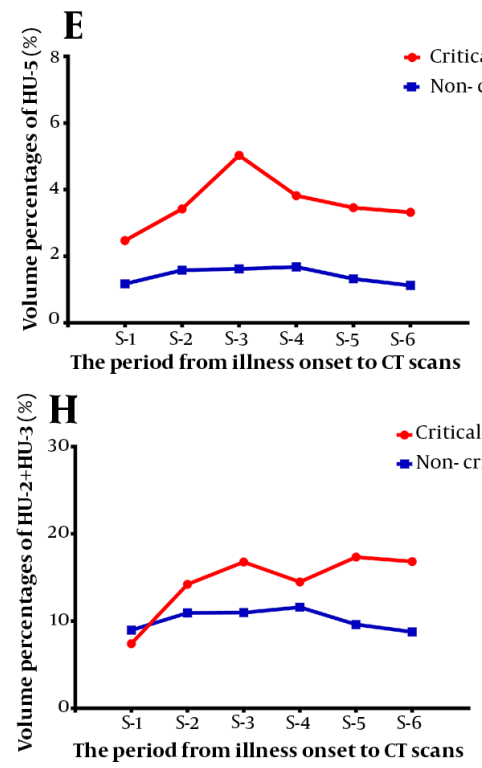

$\mathbf{F}$
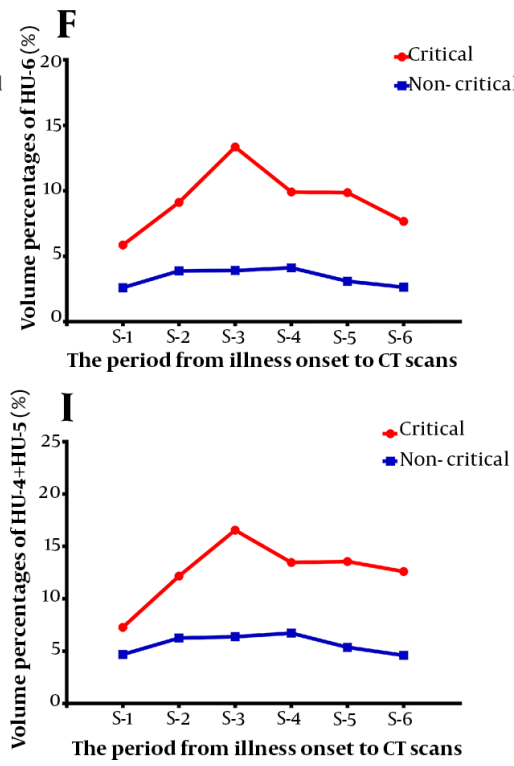

Figure 2. Temporal changes in the quantitative chest CT parameters from the time of disease onset in COVID-19 patients. This figure shows temporal changes in HU-1 (A), HU- 2 (B), HU-3 (C), HU-4 (D), HU-5 (E), HU-6 (F), HU-7 (G), HU-2+HU-3 (H), and HU-4+HU-5 (I). Differences between critical and non-critical groups were not significant in stage 1, while they were significant in all other stages, except stage 2 and stage 4 in HU-2. HU-1 to HU-7 represent the lung volume percentages in different density intervals of -1000 -700 $\mathrm{HU}(\mathrm{HU}-1),-700 \sim-600 \mathrm{HU}(\mathrm{HU}-2),-600 \sim-500 \mathrm{HU}(\mathrm{HU}-3),-500 \sim-300 \mathrm{HU}(\mathrm{HU}-4),-300 \sim-200 \mathrm{HU}(\mathrm{HU}-5),-200 \sim 60 \mathrm{HU}$ (HU-6), and $60 \sim 1000 \mathrm{HU}$ (HU-7). S-1 to S-6 represent the intervals between the disease onset and CT scan: S-1 (0 - 4 days); S-2 (5 - 9 days); S-3 (10 - 14 days); S-4 (15 - 21 days); S-5 (22 - 28 days); and S-6 (> 28 days) (COVID-19, Coronavirus disease 2019).

parameters could well assess the risk of progression to the critical stage without considering the confounders.

Some laboratory tests, such as WBC, ALT, creatinine, PCT, CK-MB, and D-dimer, did not significantly affect the relationship between most quantitative CT parameters (HU1, HU-4, and HU-7) and the severity of COVID-19. However, they could affect the relationship between some quantitative CT parameters (HU-2 and HU-3) and the severity of COVID-19. The results also showed that the lung volume percentage in HU-6 had the best predictive performance, with the largest AUC of 0.808; the cutoff value of 5.93\% showed $71.79 \%$ sensitivity and $84.62 \%$ specificity.

The present study showed that there was no significant difference between the groups regarding the lung volume percentage in different density intervals in the early stages of COVID-19 (0 - 4 days). This difference began to become significant within 5 - 9 days and persisted even after one month. It seems that changes in the quantitative CT parameters in COVID-19 patients over time could provide a reference level for distinguishing the severity of COVID-19.

This study had several limitations that need to be considered when interpreting the results. First, compared to the non-critical group, there were fewer cases in the critical group, which decreased the reliability of statistical data. Second, due to the retrospective and single-center design of this study, there may be some selection bias. Third, the CT images in this study were acquired using two CT scanners in one clinical center. Therefore, multi-center research using different scanners is necessary to validate our results. 
Table 2. Multivariable-Adjusted Associations Between Quantitative CT Parameters and COVID-19 Severity a, b

\begin{tabular}{lll}
\hline Variable & \multicolumn{1}{c}{ Crude OR $(95 \%$ CI $)$} & Adjusted OR (95\% CI ${ }^{\text {c }}$ \\
\hline HU-1 & $0.931(0.905,0.958)$ & $0.953(0.918,0.990)$ \\
HU-2 & $1.162(1.039,1.300)$ & $1.027(0.875,1.206)$ \\
\hline HU-3 & $1.404(1.199,1.646)$ & $1.157(0.933,1.436)$ \\
\hline HU-4 & $1.281(1.157,1.419)$ & $1.159(1.011,1.306)$ \\
\hline HU-5 & $1.888(1.454,2.451)$ & $1.592(1.141,2.222)$ \\
\hline HU-6 & $1.303(1.170,1.452)$ & $1.237(1.080,1.415)$ \\
\hline HU-7 & $1.999(1.453,2.750)$ & $1.768(1.114,2.808)$ \\
\hline HU-2 + HU-3 & $1.127(1.053,1.206)$ & $1.035(0.943,1.136)$ \\
\hline HU-4 + HU-5 & $1.198(1.113,1.290)$ & $1.125(1.020,1.240)$ \\
\hline
\end{tabular}

Abbreviations: OR, Odds ratio; CI, confidence interval.

${ }^{a} \mathrm{HU}-1$ to HU-7 represent the lung volume percentages in different density intervals of -1000 -700 HU(HU-1), -700 -600 HU(HU-2), -600 -500 HU(HU-3), -500 -300 $\mathrm{HU}(\mathrm{HU}-4),-300 \sim-200 \mathrm{HU}(\mathrm{HU}-5),-200 \sim 60 \mathrm{HU}(\mathrm{HU}-6)$, and $60 \sim 1000 \mathrm{HU}(\mathrm{HU}-7)$

${ }^{\mathrm{b}}$ The Hosmer-Lemeshow $(\mathrm{H}-\mathrm{L})$ goodness of fit test was used in the logistic regression model $(\mathrm{P}=0.351 \sim 0.627)$.

c Adjusted for age, sex, WBC, ALT, creatinine, PCT, CK-MB, and D-dimer.

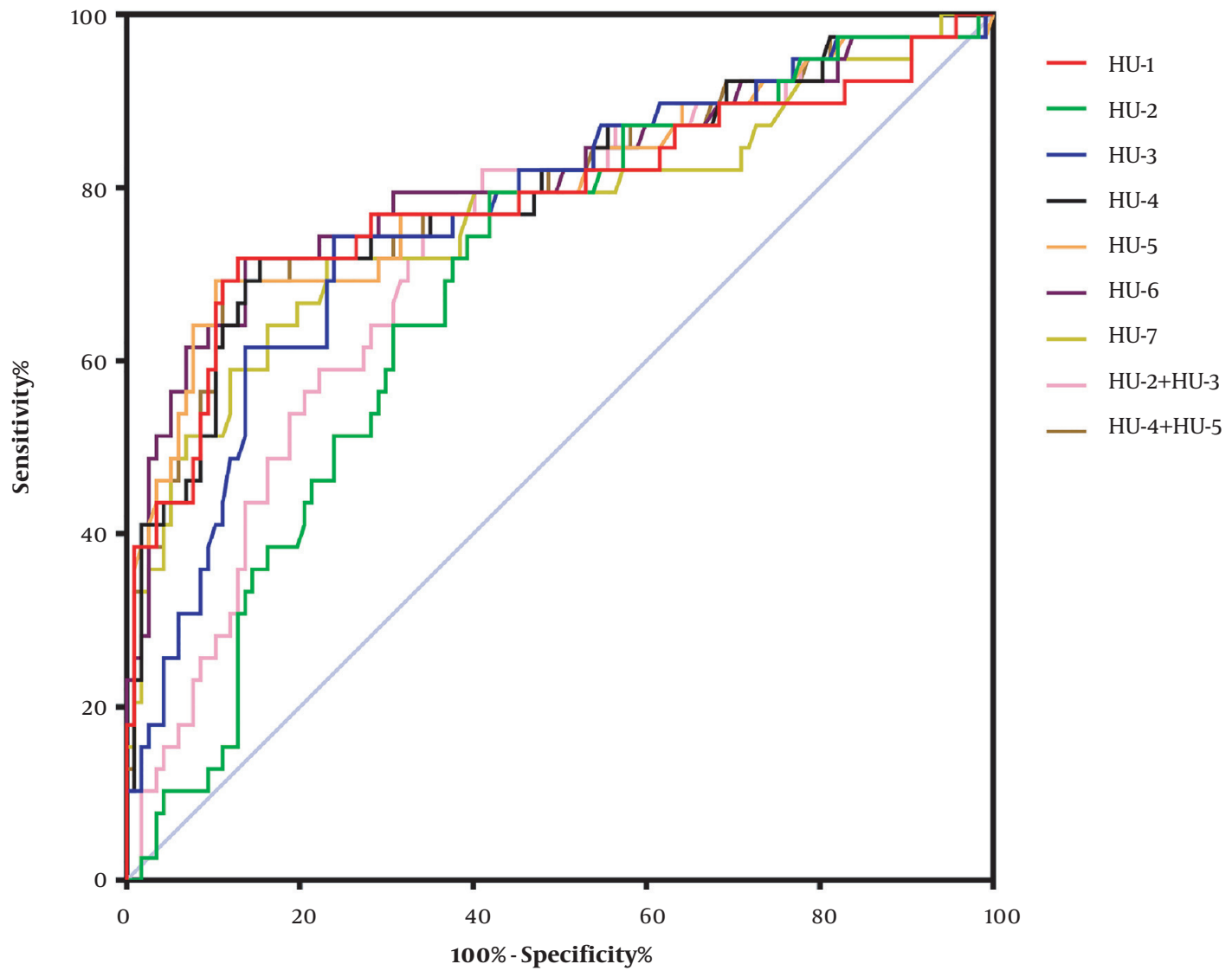

Figure 3. The ROC curve analysis of various quantitative chest CT parameters for the prediction of COVID-19 severity on admission. HU-1 to HU-7 represent the lung volume percentages in different density intervals of $-1000 \sim-700 \mathrm{HU}(\mathrm{HU}-1),-700 \sim-600 \mathrm{HU}(\mathrm{HU}-2),-600 \sim-500 \mathrm{HU}(\mathrm{HU}-3),-500 \sim-300 \mathrm{HU}(\mathrm{HU}-4),-300 \sim-200 \mathrm{HU}(\mathrm{HU}-5),-200 \sim 60$ HU (HU-6), and $60 \sim 1000 \mathrm{HU}$ (HU-7) (COVID-19, Coronavirus disease 2019; ROC curve, receiver operating characteristic curve). 


\begin{tabular}{|c|c|c|c|c|c|}
\hline Variables & $\operatorname{AUC}(95 \% \mathrm{CI})$ & Sensitivity \% (95\% CI) & Specificity \% (95\% CI) & Cutoff Value (\%) & P-Value \\
\hline HU-1 & $0.788(0.690-0.886)$ & $71.79(55.13-85)$ & $87.18(79.74-92.64)$ & 60.40 & $<0.0001$ \\
\hline HU-2 & $0.688(0.596-0.780)$ & $79.49(63.54-90.7)$ & $58.12(48.64-67.18)$ & 6.10 & 0.0005 \\
\hline HU-3 & $0.766(0.676-0.857)$ & $74.36(57.87-86.96)$ & $76.07(67.3-84.47)$ & 4.89 & $<0.0001$ \\
\hline HU-4 & $0.794(0.702-0.886)$ & $71.79(55.13-85)$ & $84.62(76.78-90.62)$ & 7.24 & $<0.0001$ \\
\hline HU-5 & $0.799(0.706-0.892)$ & $69.23(52.43-82.98)$ & $89.74(82.77-94.59)$ & 3.09 & $<0.0001$ \\
\hline HU-6 & $0.808(0.716-0.900)$ & $71.79(55.13-85)$ & $86.32(78.74$ - 91.98) & 5.93 & $<0.0001$ \\
\hline HU-7 & $0.766(0.668-0.865)$ & $71.79(55.13-85)$ & $76.92(68.23-84.21)$ & 2.82 & $<0.0001$ \\
\hline HU-2+HU-3 & $0.729(0.638-0.820)$ & $76.92(60.67-88.87)$ & $64.96(55.59-73.55)$ & 11.08 & $<0.0001$ \\
\hline HU-4+HU-5 & $0.797(0.705-0.889)$ & $69.23(52.43-82.98)$ & $88.89(81.75$ - 93.95$)$ & 11.12 & $<0.0001$ \\
\hline
\end{tabular}

Abbreviations: AUC, area under the curve; $\mathrm{CI}$, confidence interval.

${ }^{a}$ HU-1 to HU-7 represent the lung volume percentages in different density intervals of $-1000 \sim-700 \mathrm{HU}(\mathrm{HU}-1),-700 \sim-600 \mathrm{HU}(\mathrm{HU}-2),-600 \sim-500 \mathrm{HU}(\mathrm{HU}-3),-500 \sim-300$ $\mathrm{HU}(\mathrm{HU}-4),-300 \sim-200 \mathrm{HU}(\mathrm{HU}-5),-200 \sim 60 \mathrm{HU}(\mathrm{HU}-6)$, and $60 \sim 1000 \mathrm{HU}(\mathrm{HU}-7)$.

In this study, we found that quantitative chest CT parameters, classified according to different lung density intervals, could be used to assess the risk of progression to the critical stage of COVID-19. These CT parameters may be helpful for grading the severity of COVID-19 in an early stage and guide the medical staff for a better clinical management of high-risk critical cases to improve their prognosis.

\section{Footnotes}

Authors' Contribution: Study concept and design, X.D. and J.X.; Acquisition of data, X.D. and J.X.; analysis and interpretation of data, X.D., J.X. and J.Z.; Drafting of the manuscript, X.D. and J.X.; Critical revision of the manuscript for important intellectual content, Q.Y.L. and J.Z.; Statistical analysis, X.D., J.X., and J.Z.; Administrative, technical, and material support, Q.Y.L. and H.B.X.; and Study supervision, Q.Y.L., J.Z., and H.B.X.

Conflict of Interests: We declare that we have no financial and personal relationships with other people or organizations that can inappropriately influence our work.

Ethical Approval: The local ethics committee approved this retrospective study (No.: 2020037), and the need to obtain informed consent was waived.

Funding/Support: None to declare.

Informed Consent: The local ethics committee approved this retrospective study (No.: 2020037), and the need to obtain informed consent was waived.

\section{References}

1. Hong KH, Choi JP, Hong SH, Lee J, Kwon JS, Kim SM, et al. Predictors of mortality in Middle East respiratory syndrome (MERS). Thorax. 2018;73(3):286-9. doi: 10.1136/thoraxjnl-2016-209313. [PubMed: 28724637].

2. Zhou F, Yu T, Du R, Fan G, Liu Y, Liu Z, et al. Clinical course and risk factors for mortality of adult inpatients with COVID-19 in Wuhan, China: A retrospective cohort study. Lancet. 2020;395(10229):1054-62. doi: 10.1016/S0140-6736(20)30566-3. [PubMed:32171076]. [PubMed Central: PMC7270627].

3. Choi KW, Chau TN, Tsang O, Tso E, Chiu MC, Tong WL, et al. Outcomes and prognostic factors in 267 patients with severe acute respiratory syndrome in Hong Kong. Ann Intern Med. 2003;139(9):715-23. doi: 10.7326/0003-4819-139-9-200311040-00005. [PubMed: 14597455].

4. Wang D, Hu B, Hu C, Zhu F, Liu X, Zhang J, et al. Clinical characteristics of 138 hospitalized patients with 2019 novel Coronavirusinfected pneumonia in Wuhan, China. JAMA. 2020;323(11):1061-9. doi: 10.1001/jama.2020.1585. [PubMed: 32031570]. [PubMed Central: PMC7042881].

5. Guo T, Fan Y, Chen M, Wu X, Zhang L, He T, et al. Cardiovascular implications of fatal outcomes of patients with Coronavirus disease 2019 (COVID-19). JAMA Cardiol. 2020;5(7):811-8. doi: 10.1001/jamacardio.2020.1017. [PubMed: 32219356]. [PubMed Central: PMC7101506].

6. Pan F, Ye T, Sun P, Gui S, Liang B, Li L, et al. Time course of lung changes at chest CT during recovery from Coronavirus disease 2019 (COVID19). Radiology. 2020;295(3):715-21. doi: 10.1148/radiol.2020200370. [PubMed: 32053470]. [PubMed Central: PMC7233367].

7. Chung M, Bernheim A, Mei X, Zhang N, Huang M, Zeng X, et al. CT imaging features of 2019 novel Coronavirus (2019-nCoV). Radiology. 2020;295(1):202-7. doi: 10.1148/radiol.2020200230. [PubMed: 32017661]. [PubMed Central: PMC7194022].

8. Ding X, Xu J, Zhou J, Long Q. Chest CT findings of COVID-19 pneumonia by duration of symptoms. Eur J Radiol. 2020;127:109009. doi: 10.1016/j.ejrad.2020.109009. [PubMed: 32325282]. [PubMed Central: PMC7165099].

9. Pan Y, Guan H, Zhou S, Wang Y, Li Q, Zhu T, et al. Initial CT findings and temporal changes in patients with the novel Coronavirus pneumonia (2019-nCoV): A study of 63 patients in Wuhan, China. Eur Radiol.2020;30(6):3306-9. doi: 10.1007/s00330-020-06731-x. [PubMed: 32055945]. [PubMed Central: PMC7087663]. 
10. Nishiyama A, Kawata N, Yokota H, Sugiura T, Matsumura Y, Higashide T, et al. A predictive factor for patients with acute respiratory distress syndrome: CT lung volumetry of the well-aerated region as an automated method. Eur J Radiol. 2020;122:108748. doi: 10.1016/j.ejrad.2019.108748. [PubMed: 31775082].

11. Ardila D, Kiraly AP, Bharadwaj S, Choi B, Reicher JJ, Peng L, et al. Endto-end lung cancer screening with three-dimensional deep learning on low-dose chest computed tomography. NatMed.2019;25(6):954-61. doi: 10.1038/s41591-019-0447-x. [PubMed: 31110349].

12. Liu F, Zhang Q, Huang C, Shi C, Wang L, Shi N, et al. CT quantification of pneumonia lesions in early days predicts progression to severe illness in a cohort of COVID-19 patients. Theranostics. 2020;10(12):5613-
22. doi: 10.7150/thno.45985. [PubMed: 32373235]. [PubMed Central: PMC7196293].

13. Li K, Fang Y, Li W, Pan C, Qin P, Zhong Y, et al. CT image visual quantitative evaluation and clinical classification of coronavirus disease (COVID-19). Eur Radiol. 2020;30(8):4407-16. doi: 10.1007/s00330-02006817-6. [PubMed: 32215691]. [PubMed Central: PMC7095246].

14. Li L, Qin L, Xu Z, Yin Y, Wang X, Kong B, et al. Using artificial intelligence to detect COVID-19 and community-acquired pneumonia based on pulmonary CT: Evaluation of the diagnostic accuracy. Radiology. 2020;296(2):E65-71. doi: 10.1148/radiol.2020200905. [PubMed: 32191588]. [PubMed Central: PMC7233473]. 